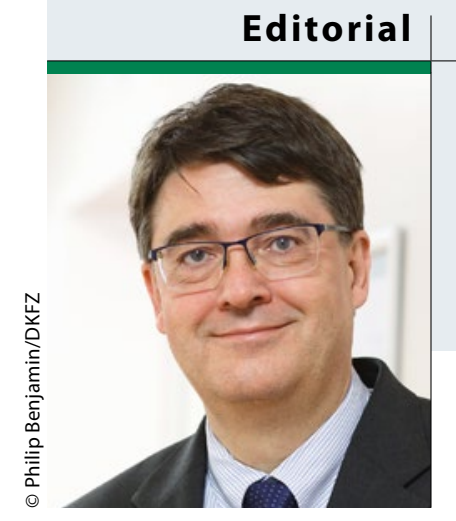

„Durch das Deutsche Konsortium für Translationale

Krebsforschung (DKTK) wird ein bislang einzigartiger Rahmen

für die personalisierte Krebsmedizin geschaffen."

Prof. Dr. med. Michael Baumann

Vorstandsvorsitzender und Wissenschaftlicher Vorstand des Deutschen Krebsforschungszentrums, Heidelberg

\title{
Konzertierte Aktion gegen Krebs
}

D ie Fortschritte hinsichtlich molekularer Tumoranalysen haben in den letzten Jahren die enorme Komplexität von Krebserkrankungen immer deutlicher zutage gebracht. Die Wechselwirkungen zwischen genetischer Veranlagung, epigenetischen Faktoren, Lebensart und Umwelteinflüssen machen diese Krankheit noch vielgestaltiger. Durch die moderne Grundlagenforschung wurden bereits viele Ansätze für individualisierte Krebstherapien aufgezeigt. Nun liegt die Herausforderung darin, die Vielversprechendsten zu identifizieren und möglichst schnell in die klinische Entwicklung zu bringen.

Um diesen Prozess zu beschleunigen, wurde im Jahr 2012 das Deutsche Konsortium für Translationale Krebsforschung, kurz DKTK, als eines der sechs Deutschen Zentren der Gesundheitsforschung gegründet. Mit dem Deutschen Krebsforschungszentrum in Heidelberg als Kernzentrum kooperieren Forscher und Ärzte an acht Standorten mit mehr als 20 akademischen Forschungseinrichtungen, darunter die von der Deutschen Krebshilfe ausgewiesenen Onkologischen Spitzenzentren. Durch diese langfristige Zusammenarbeit wird ein signifikanter Mehrwert und bislang einzigartiger Rahmen für die personalisierte Krebsmedizin geschaffen. Die Kernaufgabe des Konsortiums ist es, kontinuierlich zu prüfen, wie Ergebnisse aus der Grundlagenforschung für eine individualisierte Prävention, Diagnostik und Behandlung von Krebs genutzt werden können.

\section{Einheitliche Standards schaffen}

Um das Potenzial individualisierter Krebsmedizin breit nutzen zu können, müssen neue Verfahren und Qualitätsstandards eingeführt werden, um die an unterschiedlichen Einrichtungen erhobenen klinischen und experimentellen Tumordaten vergleichbar zu machen. Für eine Harmonisierung der Datenerhebung sorgt die Clinical Communication Plattform (CCP) des DKTK unter Einhaltung höchster Datenschutzansprüche. Wissenschaftler und Ärzte im DKTK erhalten so standortübergreifend Zugang zum klinischen Datenpool, um neue Studien und Forschungsprojekte zu planen. Der CCP angegliedert sind auch Biobanken - wahre Schatzkammern für die translationale Krebsforschung. Auch hier wird eine Vereinheitlichung von Prozessen wie Entnahme und Lagerung der Proben gewährleistet. Zudem stehen allen DKTK-Mitgliedern die Spitzentechnologien der DKTK-Standorte zur Verfügung. Dazu zählen Anlagen für die Produktion von Peptiden und Antikörpern in klinischer Qualität (Tübingen), Hochdurchsatztechnologien für die Genom- und Proteom-Analyse (Heidelberg bzw. München), bioinformatische Rechenzentren und Protonentherapieanlagen (Dresden, Essen und Heidelberg).

\section{Brücke schlagen zwischen Labor und Klinik}

Translationale Krebsforschung braucht Experten, die sowohl die naturwissenschaftliche Herangehensweise als auch die notwendige klinische Erfahrung mitbringen. Die Aus- und Weiterbildung von Ärzten und Naturwissenschaftlern ist daher ein wichtiger Fokus im DKTK. Mit der "School of Oncology“ wurde eine in Deutschland bisher einmalige Möglichkeit zur Spezialisierung in der translationalen Onkologie geschaffen. Nachwuchswissenschaftler können sich mit den Anforderungen klinischer Studien, Zulassungsprozessen und dem Konzept einer vernetzten Forschung vertraut machen. In fünf definierten Forschungsprogrammen haben sie die Möglichkeit, standort- und disziplinübergreifend Themen zu den aktuellen Herausforderungen der personalisierten Onkologie zu bearbeiten. Mit dem von Bund und Ländern langfristig geförderten Strukturaufbau für die translationale Krebsforschung vernetzen sich im DKTK einige der stärksten universitären und außeruniversitären Krebsforschungs- und Krebstherapiezentren Deutschlands mit einem gemeinsamen Ziel: die Diagnose und Behandlung von Krebs schnellstmöglich und nachhaltig zu verbessern.

Michael Baumann 